\title{
Organizational Challenges in Developing One of the Nationwide Health Information Network Trial Implementation Awardees
}

\author{
Aram Dobalian • Maria L. Claver • Joshua M. Pevnick • \\ Harris R. Stutman • Alan Tomines • Paul Fu Jr.
}

Received: 26 February 2010 / Accepted: 5 July 2010 /Published online: 20 July 2010

(C) The Author(s) 2010. This article is published with open access at Springerlink.com

\begin{abstract}
Health care in the United States is rarely delivered in a coordinated manner. Current methods to share patient information are inefficient and may lead to medical errors, higher readmission rates, and delays in the delivery of needed health services. This qualitative study describes lessons learned concerning the early implementation of one Nationwide Health Information Network (NHIN) site in Long Beach, CA during its first year of operation. The Long Beach Network for Health (LBNH) focused on an incremental effort to exchange health information. Despite a limited concentration on emergency department care, virtually all respondents noted concerns regarding the sustainability, or business case, for the exchange of health information. Nevertheless, respondents were encouraged by progress on technological challenges and user requirements during this first year. The early gains in this process may, in turn, have laid the groundwork for future efforts to expand beyond the emergency department.
\end{abstract}

A. Dobalian $(\bowtie) \cdot$ M. L. Claver

Department of Veterans Affairs Greater Los Angeles

Healthcare System, HSR\&D Center for the Study of Healthcare

Provider Behavior,

16111 Plummer St. (152),

Sepulveda, CA 91343, USA

e-mail: aram.dobalian@va.gov

A. Dobalian · A. Tomines $\cdot$ P. Fu Jr.

Department of Health Services,

UCLA School of Public Health,

Los Angeles, CA, USA

\section{L. Claver}

Department of Family \& Consumer Sciences,

California State University,

Long Beach, CA, USA
Keywords Emergency medical services $\cdot$ Health informatics $\cdot$ Medical record, electronic $\cdot$ Organizational systems $\cdot$ Technology $\cdot$ Medical

\section{Introduction}

The delivery of health services in the United States rarely occurs in a coordinated manner, in part due to challenges related to sharing patients' health information between different healthcare providers. To assemble a more complete patient medical history, providers have traditionally shared entire paper records or short excerpts of such records via fax, courier, or other means. These methods are inefficient, and may fail to ensure that providers have access to needed health information in a timely fashion. These inefficiencies may lead providers to repeat tests or procedures rather than obtain paper records from other
J. M. Pevnick

Division of General Internal Medicine, Department of Medicine,

Cedars-Sinai Medical Center,

Los Angeles, CA, USA

H. R. Stutman

Miller Children's Hospital, MemorialCare Health System,

Long Beach, CA, USA

A. Tomines $\cdot$ P. Fu Jr.

Division of General and Emergency Pediatrics,

Harbor-UCLA Medical Center,

Torrance, CA, USA

A. Tomines $\cdot \mathrm{P} . \mathrm{Fu} \mathrm{Jr}$.

Los Angeles Biomedical Research Institute,

Torrance, CA, USA 
providers. The resulting duplication of services increases the cost of delivering healthcare in an already expensive healthcare system. In addition, the inefficiencies and inaccuracies inherent in sharing paper records may negatively impact quality of care by leading to additional opportunities for medical errors, higher readmission rates, and delays in the delivery of needed health services [1].

Thus, there is a need to coordinate the sharing of patients' medical records and other clinical information obtained from different healthcare organizations into an easily-accessible system that integrates diverse, stand-alone private health information systems regardless of whether the records are in paper or incompatible electronic formats. An electronic network would potentially facilitate the exchange and integration of clinical information as well as administrative and claims data [1]. It has been suggested that the implementation of such a health information exchange system on a national scale could lead to significant cost savings, perhaps as much as $\$ 77.8$ billion per year, although the initial cost of implementation may be substantial [2].

Despite the potential for cost-savings and improvements in quality of care, significant financial, technological, and policy barriers impede the development of such a system. For example, providers who might use such a system may be competitors in a given market, and aligning health information services with the needs of each competitor is difficult. Competing interests and concerns regarding the costs of systems for sharing health information across providers have delayed their widespread adoption. In addition, many healthcare facilities lack electronic medical record systems. Adding such systems imposes additional front end costs on providers, in addition to the challenges and costs associated with sharing information.

The Office of the National Coordinator (ONC) is responsible for providing "leadership for the development and nationwide implementation of an interoperable health information technology infrastructure to improve the quality and efficiency of health care [3]." ONC is charged with leading efforts to implement a nationwide network by 2014.

In October 2007, \$22.5 million was awarded to nine organizations across the nation to pilot Health Information Exchanges (HIEs) to share health information electronically within and among HIEs [4]. These pilot HIEs would test various architectures for data exchange and serve as models of organization, technical infrastructure, governance and operational sustainability for other nascent HIEs across the country. Once fully operational, these interconnected HIEs would constitute the initial backbone for the Nationwide Health Information Network (NHIN).

The pilot HIE within Los Angeles County is the Long Beach Network for Health (LBNH). Created in 2003,
LBNH started as a public-private coalition of local stakeholders with the goal of establishing an organizational framework for HIE. An interest-free loan of $\$ 100,000$ from one of these stakeholders provided the initial funding for LBNH. LBNH incorporated as a non-profit corporation in 2007. A five-member Executive Committee oversees the day-to-day operations of LBNH. Stakeholders participating in the LBNH NHIN provided in-kind services in support of the HIE's initial efforts.

LBNH's initial efforts focused on the city of Long Beach, in preparation for a larger expansion across Los Angeles County. LBNH offers an opportunity to examine the factors that influence the implementation of an HIE that serves an urban, poorer population with a relatively high number of uninsured people. While the lack of health insurance and underinsurance affect the financial standing of most, if not all, healthcare institutions across the country, these issues have been particularly severe and longstanding in Southern California. Recently, the region has also confronted the closure of a major county hospital in an underserved area as well as planned Medi-Cal payment reductions to providers. The city of Long Beach has a population of over 463,000 , and a median per capita income of $\$ 24,021$, which is low by state standards [5]. Long Beach has 5 hospitals (including 2 trauma centers), and more than 2,300 practicing physicians. In comparison with similar efforts in other urban settings, LBNH is developing its HIE with fewer resources.

LBNH initially planned to address the goals of the NHIN by reporting data for public health purposes and supporting safety-net providers. However, local public health agencies lacked sufficient funding to support the initial infrastructure investments necessary for LBNH. LBNH subsequently refocused their efforts on an Emergency Department (ED) linkage project to make critical inpatient and outpatient information available to ED clinicians. Three hospitals and two ambulatory practice groups in Long Beach provided the clinical data electronically to the EDs of the three participating hospitals in Long Beach. LBNH is using a turnkey FirstGateways exchange solution platform based on a hybrid federated standards-based architecture, and includes a customizable web application (HealthView ${ }^{\mathrm{TM}}$ ), back office services (MPI, RLS, security), integration/data mining services, and a library of commercial interfaces (i.e., a commercial HIE infrastructure and clinical portal/data exchange engine).

Emergency physicians are often required to make decisions without sufficient access to historical patient information. It has been suggested that better access to patient data at the point of care may improve the quality of care provided within EDs and potentially alleviate overcrowding [6]. A prior study by Shapiro, Kannry, Kushniruk, and Kuperman [7] found a strong perceived need for health 
information exchange among physicians in EDs. LBNH's focus on EDs in its first-year allows us to examine the roll-out of an HIE that meets an immediate need of providers and therefore has the potential to build trust in the community. A small success such as this may be important prior to a larger programmatic expansion, as it may curtail the kind of mistrust among providers that led to the failure of prior efforts at the exchange of health information $[8,9]$.

This study describes the LBNH HIE during its first year of operation (2008), and in so doing, provides lessons learned in the implementation of an HIE that focused on an incremental effort to exchange health information. Representatives from local institutions provided information about their organization's efforts to implement the data exchange in conjunction with LBNH, and described organizational characteristics that may have played a role in each organization's decision to participate. During the time of this study, LBNH successfully exchanged test data, but had not exchanged actual patient care data. Information regarding the status of other HIEs across the nation is available from the eHealth Initiative, which conducts an annual survey on HIE [10], and in a survey of 145 regional health information organizations (RHIOs) conducted by Adler-Milstein and colleagues [11]. Per the 2008 eHealth Initiative survey, there were 42 operational HIE initiatives.

\section{Materials and methods}

The focus of this project is to assess the progress of the LBNH HIE during its first year. We conducted interviews with key respondents from organizations that both participate and do not participate in LBNH using a semistructured interview protocol. The interview protocol included a general set of questions for all participants and a secondary set of questions that addressed issues particular to each participant's organizational role (such as such as Chief Executive Officer, Chief Information Officer, or Chief Medical Officer). Copies of the interview guides are available elsewhere [12]. No interview took longer than $90 \mathrm{~min}$, and many aspects were adequately studied in $30 \mathrm{~min}$. Interviews were semi-structured and conducted by telephone.

The interviews followed well-established procedures for conducting semi-structured interviews [13]. Within each topic area of the interview protocol, open-ended questions typically were asked before closed-ended questions so as not to bias a respondent's answers. An initial open-ended format allows both the interviewer and the respondent the opportunity to explore new leads and related topics and can often generate rich personal narratives. Answers to such questions are indicative of areas that are most important to respondents - and may serve to confirm or disconfirm our expectations. We also used standard probes, such as verification and compare-and-contrast questions, to elicit additional information [14].

All interviews were conducted by at least two members of the research team, with one interviewer taking the responsibility for leading interviews and the other person taking detailed notes. Other investigators led some interviews after being trained and participating in a number of interviews as note-takers. The interviews were audio recorded with the participants' permission. Transcriptions of the interviews and notes taken during the interview were reviewed during subsequent data collection to identify key themes in the textual data. To deduce themes from the textual data, the team read through all interview notes and looked for examples that suggested processes, actions, assumptions, and consequences. Themes were abstracted from the interview notes and organized into a matrix of coding categories. The analysis aggregated overall themes, and compared and contrasted those themes across sites, where differences among settings became apparent. After separately examining the data, the team came to agreement about which themes to examine in more detail.

Interviews were conducted with representatives from LBNH and nine stakeholder organizations including: a network of several community health centers; a local notfor-profit health maintenance organization; two multispecialty physician medical groups; a regional independent practice association; a large community hospital that is a member of a local not-for-profit healthcare system; a large community hospital that is a member of a large regional not-for-profit healthcare system; a publicly-traded managed healthcare company operating in several states; and a teaching hospital that operates under the purview of the local municipal government. The five institutions that exchanged clinical data electronically during the period of this study included one of the two multispecialty physician medical groups, the regional independent practice association, both large community hospitals, and the teaching hospital.

Within each of these 10 institutions, we attempted to interview at least one of the following: HIE champion, chief executive officer (CEO), chief information officer (CIO), or chief medical officer (CMO). Interview content addressed the process of learning about LBNH, the institution's decision whether to participate in LBNH's data sharing network, early interactions with LBNH, barriers and facilitators to implementation of the NHIN, and an overall assessment of the NHIN's progress during the first year. LBNH leadership was also interviewed to evaluate interactions between LBNH and the healthcare institutions from multiple perspectives. 


\section{Results}

Eighteen interviews were scheduled and conducted with representatives from ten organizations. The most common position of an interviewee was $\mathrm{CEO}$, which accounted for four of the 18 respondents. The next most common position was CIO, which represented three of the 18 respondents. Two CMIOs were interviewed. Other respondents had various titles; several were attorneys who functioned in one of the previously-identified executive roles with their respective organizations. Respondents with legal training often addressed compliance and privacy issues during the course of the interviews. We interviewed multiple individuals at most organizations, allowing us to assess differences in interviewee responses.

\section{Participation in the LBNH HIE: Organizational decision-making processes and factors}

There were two main approaches to decision-making that organizations used in determining the nature and extent of their participation in the LBNH HIE: (a) a formal structure for decision-making, and (b) designating an organizational representative. Some respondents also identified additional factors that may influence their organization's decision to participate.

\section{Formal structure}

Some participants described a formal decision-making structure such as an organizational planning committee, which outlined the mission, goals, and availability of funding for the organization to participate in LBNH. One respondent mentioned an IT committee as the main decision-making body at his organization, while another described an "executive team" responsible for decisions regarding participation in health information exchange efforts. Several mentioned that a more formal decision-making process or excessive bureaucracy could lead to lengthy delays, particularly when decisions concerned privacy considerations related to the Health Insurance Portability and Accountability Act (HIPAA). In general, respondents from larger organizations tended to have more formal decision-making processes, whereas respondents from smaller organizations noted that individuals were given more latitude.

\section{Organizational representative}

While many of the respondents described a formal decisionmaking process regarding whether to participate in health information exchange through LBNH, others mentioned that they were asked by their supervisor to participate in LBNH as a representative of their organizations. One respondent specifically mentioned that he thought it would be more effective to participate on the LBNH board in an individual capacity rather than as a representative from a particular organization, because obtaining approval from the respondent's supervisors would cause considerable delays in the decision-making process. This respondent preferred that participants in the LBNH planning and decision-making process either operate independently from their employer, or that the place of employment should empower the representative with decision-making authority.

Other factors relevant to participation

Additional factors that influenced various organizations' decision-making about participation in health information exchange included: (a) whether the HIE was likely to be self-sufficient in terms of funding without seed money and grants, (b) whether the organization had a physician champion who would encourage participation from physicians, and (c) whether participation in an HIE fit the strategic plan of the organization, such as a focus on the public health of the community.

\section{Initial awareness and level of interaction with LBNH}

Respondents became aware of LBNH in one of three ways: (a) learning about LBNH by following health information exchange efforts in general, (b) knowing someone or working with colleagues who became involved with LBNH, and (c) being invited to participate on the planning committee.

Following health information exchange efforts in general

One participant mentioned that he had been reading about health information exchange efforts across the country for several years before learning about LBNH. Another mentioned that he had a personal interest in health information exchange, and therefore actively sought out information about collaborations forming around this topic.

\section{Knowing someone involved in LBNH}

Several participants mentioned that they were introduced to LBNH through the Long Beach Department of Public Health or knew someone already on the LBNH planning committee. One respondent said that he learned about LBNH when the information technology and finance departments at his place of employment became involved and shared the information with him. 
Invitation to participate

Several respondents were invited to participate in the planning committee of LBNH either directly by LBNH, or were delegated the position by a supervisor who was invited to participate by LBNH leadership.

\section{Barriers/challenges to implementation}

Barriers mentioned by the respondents fell into one of the following nine categories: (a) financial resources, (b) patient privacy and concerns about misuse of data, (c) industry competition, (d) infrastructure, (e) lack of a business case, (f) leadership, (g) competing priorities, (h) training, and (i) interoperability with existing systems.

\section{Financial resources}

Many of the respondents were concerned about the financial resources that would be necessary to either set up the infrastructure to support the NHIN or to alter existing technology necessary to participate in HIE, especially during a time when cost-cutting has become a priority.

\section{Patient privacy and misuse of data}

Patient privacy and confidentiality, especially for vulnerable populations such as persons living with HIV, persons with psychological disorders, or celebrity patients, were mentioned as a challenge to implementation of HIE. Many respondents mentioned concerns about conforming to HIPAA rules, and noted that their organization's legal departments were examining these considerations. While a few participants hoped the data that are exchanged could be used for public health purposes, one mentioned a desire to be assured that it would not be used for that purpose, or for research or marketing, as those uses would not be included in patients' current HIPAA authorizations. This respondent also noted that the organization's perspective is that the data is being collected for clinical purposes and should be used solely for clinical purposes. Another respondent identified limitation was that the data might not be sufficiently granular to be used meaningfully for research. One respondent noted that as the number of individuals who have access to the data expands, the likelihood that an unauthorized individual may access patient data increases. This respondent expressed particular concern that such unauthorized use of data may occur when the HIE expands to include sharing data with non-ED settings. Regarding data security, multiple respondents also acknowledged the potential for bad publicity in the event of unauthorized access to data.
Industry competition

Respondents shared that colleagues at other institutions had indicated some concern about losing patients as an indirect consequence of data sharing, proposing it would be easier for a patient to change from healthcare provider to provider if patient information were readily available. Although respondents raised this issue as being of concern for their colleagues, when asked to share their perspective on the issue of industry competition, they did not endorse this view as being true to their own experience. Some respondents also noted that others had expressed concern regarding the possibility that the availability of patient data, including treatment plans, could give providers at other participating organizations ammunition to decide that care was "substandard." This concern, in conjunction with haphazard comments by those accessing these data, could potentially lead to malpractice claims, negatively affect reimbursement rates based on quality of care, or result in the loss of patients to other providers.

\section{Infrastructure}

Respondents were concerned about how the technical aspects of how HIE would work, but expressed the belief that these technical considerations would not preclude adoption of HIE. One respondent wondered how data would be retrieved from her agency's database and be transmitted to another database when requested. Another felt that HIE was an untested practice and that this could be a barrier to implementation. Another potential challenge mentioned included the belief that proponents of health information systems often underestimate the complexity inherent in actual implementation. Lastly, many organizations noted that numerous providers do not yet have the electronic medical records necessary to populate an HIE, and this was thought to limit the capacity of such organizations to participate in the NHIN.

\section{Business case/model and leadership}

Several of the respondents stated that strong leadership and a clear business model were necessary for a health information exchange project to move forward and be successful. One respondent felt that having a board comprised of individuals rather than hospitals would provide appropriate flexibility to debate strategies, goals and tactics without having to wait for authorizations from hospital administration. Another stated that including government entities in the leadership and decision-making process would slow down progress toward implementation of the NHIN because of the bureaucratic nature of these agencies. Several respondents stated that obtaining a critical mass of participants was necessary to move this project forward. 


\section{Competing priorities}

For some of the respondents' organizations, participation in the exchange of health information was not viewed as a priority for the organization. One respondent said, "If it is a number 25 item for the folks in the information services department, it is probably number 125 for the hospital as a whole. I just don't think it is a high visibility item for them where they say, 'This is wonderful. We are going to get better relationships with our key medical groups.' At this point in time right now, I would say [it is] more of a distraction in that we have a project list that probably has $50-100$ projects on it. Some of it is more direct patient care..., infrastructure, [and] upgrading PCs." According to respondents from organizations for whom participation in the exchange of health information is not a priority, some providers do not believe that the sharing of patient data in electronic form is critical for patient care.

\section{Training}

Assuming the technological infrastructure for HIE is functioning well, the next challenge to incorporating HIE data into regular ED practice is training providers to use the technology. Respondents stated that it was difficult for busy emergency care providers to find time to attend trainings on how to use HIE. Moreover, some respondents stated that, in particular, medical assistants who perform administrative and certain clinical duties under the direction of a physician were not usually technologically savvy, and that training would need to be prioritized for this group to a greater extent than for other providers.

\section{Data definitions}

The last challenge identified by the respondents in this study was interoperability itself. Agencies sharing data may define information differently and it is possible that data will therefore not translate correctly across systems. This aspect of HIE would have to be confronted before data sharing could take place. None of the respondents indicated that this challenge was insurmountable.

\section{Overall assessment of progress during the first year}

On a positive note, some respondents felt that LBNH was more concerned with actually exchanging data compared with other data exchange organizations they had worked with in the past. Furthermore, many respondents cited the "incredible potential" of LBNH given the value of the data of participating organizations. One respondent said that the progress of HIE has gone as fast as was feasible, and noted that there has been proof that data exchange is feasible from other HIEs and RHIOs. This respondent further indicated that at this point, the challenges concern selling the product, proving it functions, demonstrating its utility, and inspiring other providers to participate.

Not all respondents were as encouraged by the efforts of LBNH during the first year. Some respondents noted lingering concerns that there has been a lower than expected return on their investment thus far, and that although LBNH has received some favorable publicity, health information exchange has had a low impact on the delivery of care within participating hospitals. One said, "I don't think we have seen enough coming out of this process that says to us, okay, someone has the secret sauce here," despite the efforts of LBNH and others. Respondents acknowledged issues regarding delays in the implementation of the HIE, data ownership and structure, and perceived fairness of everyone sharing equal amounts of data. Nonetheless, most respondents stated that this project will expand and mature with additional incentives from the federal government, and stated that for LBNH to succeed, it must become a part of the ED culture.

\section{Discussion}

LBNH began as a local public-private coalition of stakeholders in Long Beach and Los Angeles. Its goal was to establish an initial organizational framework for health information exchange. To date, LBNH is the only HIE to become a pilot for the NHIN on the west coast. LBNH's focus in the first year of the NHIN contract has been on the development of a hardware and software platform that could deliver critical patient information to healthcare professionals in the EDs of Long Beach Memorial Medical Center, Miller Children's Hospital, and Los Angeles County Harbor - UCLA Medical Center.

Many of the challenges noted by our respondents have been identified by others [7, 8, 15-18], although a concern that the data not be used for research purposes is of particular note. The promise of HIE is, in part, predicated on the assumption that access to widespread community data will permit the examination of complex clinical research questions that would be difficult or impossible to address through alternative methodological approaches. This potential presupposes careful consideration of the balance between patient privacy and confidentiality and the potential societal benefit. Deidentification of the data would address this concern in many instances, but may limit the potential societal benefit, such as, for example, in the case of relatively rare conditions that cannot be readily deidentified. One question related to patient privacy not captured by this study is an assessment of the effect of emergency medical records on patient-physician interaction [19]. 
Fear of losing patients to competitors was not a significant concern among our respondents, in contrast to some prior studies [20]. This may relate to a lack of competition for certain patient populations in the catchment area of the LBNH participants. For example, patients that use the services of the Harbor-UCLA Medical Center are more likely to be uninsured than the general population. Similarly, patients that visit Miller Children's Hospital may be unlikely to seek treatment from other children's hospitals that are many miles away.

The infrastructure concerns expressed by some respondents are not unique to HIE. While it would be important to consider, for example, access to an adequate number of workstations, these technical challenges need not necessarily preclude an organization's participation. Rather, an organization could plan for a roll-out of HIE and address these concerns as they arise, provided that the additional expense associated with the roll-out is not excessive.

Despite the current focus of LBNH on ED care, virtually all respondents noted concerns regarding the sustainability, or business case, for HIE. Sustainability is a common concern among RHIOs, but little empirical data exists on the subject [11]. This finding is of some concern as the ED is often discussed as having significant potential to benefit from HIE [7]. This may reflect the fact that LBNH is a work in progress and additional work remains to be completed to share data more widely. As LBNH progresses and more data are made available, respondents may see greater benefits to sharing data in EDs. Other advantages of this "radical incrementalism" approach [8, 18, 21-23], which in the case of LBNH, starts with the exchange of ED data, are better supported by our findings. As defined by Frolich and colleagues [8], "radical incrementalism" typically involves rapid 6-month to 12-month initiatives, in support of a longerterm strategic direction. Although each successive small wave represents a step toward this direction, the cumulative effect may be radical change. For example, respondents did note that many technological challenges, user requirements, and other concerns had been addressed at least in part during this first year. These respondents were encouraged by this progress.

Also of note is the skepticism expressed by some respondents as to the value of HIE, and its importance relative to other organizational considerations or individual responsibilities. In part, this may reflect the decentralized decision-making process in most participating organizations and a consequent lack of buy-in for the LBNH NHIN as we moved down the chain of command from the CEOs to other respondents in each organization. Nonetheless, many respondents viewed their participation in the LBNH NHIN as central to their organization's mission of providing care for the community.

It is possible that LBNH can build on its technical progress during the first year to gain additional support from local healthcare providers. Our interviews suggest, however, that additional support may also require the development of a stronger business case for HIE in the ED and demonstration of improvements in quality of care for ED patients.

The ultimate success or failure of the LBNH NHIN is uncertain at this time. Substantial concerns persist regarding the optimal and appropriate use of the data. Nonetheless, there are encouraging signs that suggest that it may ultimately be successful. Despite lingering concerns regarding privacy, data security, and sustainability of the business model, most respondents were encouraged by the progress made by LBNH during this early phase. The early gains in this process may, in turn, have laid the groundwork for future efforts to expand beyond the ED. In this regard, LBNH provides some early indication that a model aimed at a series of small, successful initiatives may ultimately lead to a larger adoption of HIE, albeit over the longerterm, as others have suggested [8]. This incremental approach may also be more feasible for communities that lack comparable financial support to that available to other HIE efforts, such as the Health Information Technology Evaluation Collaborative (HITEC) in New York [16].

\section{Conclusion}

Despite the concerns we heard from some respondents, it seems likely that the U.S. will continue to move toward widespread adoption of HIE because of the passage of the Health Information Technology for Economic and Clinical Health (HITECH) component of the American Recovery and Reinvestment Act of 2009. HITECH is intended to promote the widespread adoption of health information technology, and includes funding targeted at the implementation of HIE.

The widespread use of health information technology is perceived to be critical to solving the challenges confronting the U.S. healthcare system, whether by reducing medical errors, improving healthcare quality, or reducing expenditures associated with inappropriate use of duplicative health services. HIEs, when properly supported, funded, and implemented based on the needs of their particular local communities, offer the potential to securely exchange patient data beyond traditional organizational boundaries to address these challenges. Furthermore, access to HIE data can potentially be used for quality assessment/ improvement and biosurveillance at a lower cost and to a further degree than any comparable system.

Acknowledgement This work was supported by DHHS Contract HHSP23320074104EC. The views expressed in this article are those of the authors and do not necessarily reflect the position or policy of the Department of Veterans Affairs or the United States government. 
Open Access This article is distributed under the terms of the Creative Commons Attribution Noncommercial License which permits any noncommercial use, distribution, and reproduction in any medium, provided the original author(s) and source are credited.

\section{References}

1. Brailer D, Nikolas Augustinos, Lori Evans, and Sam Karp. Moving Toward Health Information Exchange. Interim Report on Santa Barbara County Care Data Exchange: California Healthcare Foundation; July 2003.

2. Walker J, Pan E, Johnston D, Adler-Milstein J, Bates DW, Middleton B. The value of health care information exchange and interoperability. Health Aff. (Millwood). Suppl Web Exclusives: W5-10-W5-8, 2005.

3. The ONC Coordinated Federal Health IT Strategic Plan: 200820012. January 2008. (Accessed June 24, 2010, at http://www. hhs.gov/healthit/resources/HITStrategicPlan.pdf.)

4. HHS Awards Contracts for Trial Implementations of the Nationwide Health Information Network. October 5, 2007. (Accessed June 24, 2010, at http://www.hhs.gov/news/press/2007pres/10/ pr20071005a.html.)

5. 2005-2007 American Community Survey. (Accessed April 22, 2009, at http://www.census.gov/acs/www/index.html.)

6. Institute of Medicine, Hospital-based emergency care: At the breaking point. National Academy, Washington, DC, 2006.

7. Shapiro, J. S., Kannry, J., Kushniruk, A. W., and Kuperman, G., Emergency physicians' perceptions of health information exchange. J Am Med Inform Assoc 14:700-5, 2007.

8. Frohlich, J., Karp, S., Smith, M. D., and Sujansky, W., Retrospective: Lessons learned from the Santa Barbara project and their implications for health information exchange. Health Aff (Millwood) 26:w589-91, 2007.

9. Garets, D. E., Why RHIOs aren't working: Views from an American who can see White Rock, British Columbia, from his backyard. Healthc Q 11:102-3, 2008.

10. eHealth Initiative Launches 2010 Survey on Health Information Exchange. 2010. (Accessed June 24, 2010, at http://www. ehealthinitiative.org/ehealth-initiative-launches-2010-surveyhealth-information-exchange.html.)

11. Adler-Milstein, J., McAfee, A. P., Bates, D. W., and Jha, A. K., The state of regional health information organizations: Current activities and financing. Health Aff (Millwood) 27:w60-9, 2008.

12. Pevnick JM, Claver M, Dobalian A et al. Provider stakeholders' perceived benefit from a nascent health information exchange: A qualitative analysis. J. Med. Sys. 2010.

13. Patton, M. Q., Qualitative evaluation and research methods, 2nd edition. Sage, Newbury Park, 1990.

14. Bernard, H. R., Research methods in anthropology: Qualitative and quantitative approaches, 3rd edition. AltaMira, Walnut Creek, 2002.

15. Johnson, K. B., and Gadd, C., Playing smallball: Approaches to evaluating pilot health information exchange systems. $J$ Biomed Inform 40:S21-6, 2007.

16. Kern, L. M., and Kaushal, R., Health information technology and health information exchange in New York State: New initiatives in implementation and evaluation. J Biomed Inform 40:S17-20, 2007.

17. Protti, D., US regional health information organizations and the nationwide health information network: Any lessons for Canadians? Healthc $Q$ 11:96-101, 2008. 4.

18. Stoop, A. P., and Berg, M., Integrating quantitative and qualitative methods in patient care information system evaluation: Guidance for the organizational decision maker. Methods Inf Med 42:45862, 2003.

19. Rhodes, P., Small, N., Rowley, E., Langdon, M., Ariss, S., and Wright, J., Electronic medical records in diabetes consultations: Participants' gaze as an interactional resource. Qual Health Res 18:1247-63, 2008.

20. Grossman JM, Kushner KL, November EA. Creating sustainable local health information exchanges: Can barriers to stakeholder participation be overcome? Res. Briefs. 1-12, 2008.

21. Friedman, C. P., "Smallball" evaluation: A prescription for studying community-based information interventions. J Med Libr Assoc 93:S43-8, 2005.

22. Kaplan, B., and Shaw, N. T., Future directions in evaluation research: People, organizational, and social issues. Methods Inf Med 43:215-31, 2004.

23. Stead, W. W., Haynes, R. B., Fuller, S., et al., Designing medical informatics research and library-resource projects to increase what is learned. J Am Med Inform Assoc 1:28-33, 1994. 